associated with a photoparoxysmal response to intermittent photic stimulation. In total, 610 patients participated in the study; $65 \%$ were children, $68 \%$ had generalized epilepsy, and $66 \%$ were female. At the start of the study, $62 \%$ of patients were taking antiepileptic drugs. A standardized method of photostimulation was performed on patients in a darkened room, both with and without the lens, and split-screen video electroencephalogram was used to record the events. Statistical analysis was used to assess potential variations in the response caused by baseline differences among patients.

In $75.9 \%$ of patients the $\mathrm{Z} 1$ lens resulted in the disappearance of the photoparoxysmal response, and in $17.9 \%$ of patients the photoparoxysmal response was reduced. Patients' age, sex or epilepsy type had no significant influence on the type of photoparoxysmal response to the lens, and there was no difference in lens efficacy between patients receiving antiepileptic drugs and those not receiving pharmacological treatment.

Concluding that the Z1 lens is highly effective in controlling the photoparoxysmal response in patients with epilepsy, the authors suggest that it could become a valid treatment option for the condition.

Original article Capovilla G et al. (2006) Suppressive efficacy by a commercially available blue lens on PPR in 610 photosensitive epilepsy patients. Epilepsia 47: 529-533

\section{Mutations in COL4A1 might increase risk of hemorrhagic stroke}

Cerebral small-vessel disease is associated with intracerebral hemorrhage and ischemic stroke. Gould et al. have identified mutations in the gene encoding type 4 collagen $\alpha 1$, a basement membrane protein, that cause vascular disease in both humans and mice.

Mice carrying mutations in the Col4a1 gene were predisposed to stress-induced cerebral hemorrhage. All naturally born newborn mutant mice had hemorrhage due to birth trauma, and approximately $50 \%$ of these pups died on the day of birth. All adult mutant mice studied had intracerebral hemorrhage, and the death rate was higher in mutant mice than in wild-type mice. Some mutant mice were also affected by retinal vascular tortuosity, glomerular basement membrane defects and microalbuminuria.
Phenotypic similarities between mice with Col4a1 mutations and a French family with smallvessel disease led the researchers to screen the family for COL4A1 mutations. Family members were affected by retinal arteriolar tortuosity, MRI scans showed cerebral microbleeds and whitematter abnormalities, and two family members died of intracerebral hemorrhage. Sequence analysis revealed a point mutation in COL4A1 that caused a glycine residue in the triple-helix domain of the protein to be replaced by glutamic acid. Glycine residues are highly conserved in type 4 collagen $\alpha 1$, and mutations that affect these residues are known to be pathogenic in many species.

The authors conclude that COL4A1 mutations cause weakening of the vascular basement membrane and predispose affected individuals to hemorrhage, and suggest that mutations in other basement membrane proteins could also cause small-vessel disease.

Original article Gould DB et al. (2006) Role of COL4A1 in small-vessel disease and hemorrhagic stroke. N Engl J Med 354: 1489-1496

\section{Transcranial direct current stimulation controls pain in traumatic spinal cord injury}

Pain associated with brain or spinal cord lesions is often refractory to drug treatment. A number of cortical stimulation techniques have been developed for the treatment of pain associated with these conditions. Among these, transcranial direct current stimulation (tDCS) offers some advantages, as it is a simple and inexpensive technique that uses a weak direct electric current to stimulate the cerebral cortex noninvasively. In a sham-controlled, phase II trial, Fregni et al. evaluated the effect of treatment with tDCS in patients with refractory central pain.

Eleven patients received active tDCS, which consisted of a $2 \mathrm{~mA}$ current applied to the primary motor cortex for 20 min on five consecutive days. Six patients received sham treatment. Patients were classed as responders to treatment if they experienced a reduction of $50 \%$ or more in their pain score as measured by a $10 \mathrm{~cm}$ visual analog scale (VAS). There were seven responders in the intervention group and one in the control group after the completion of treatment. The mean VAS score in the intervention group fell from 6.2 to 2.6, 